\title{
Audit
}

\section{Assessment of head and neck cancer care in District General Hospital Trincomalee}

Ekanayake LSB ${ }^{1}$ D, Vasantharajah $\mathrm{G}^{2}$, Alex $\mathrm{PA}^{2}$, PriyadarshaniK ${ }^{2}$,Dharshi MKS ${ }^{2}$,Dilrukshi MKN².

${ }^{1}$ Consultant ENT and Head \& Neck Surgeon, DGH Trincomalee, Sri Lanka

${ }^{2}$ Medical Officer ENT, DGH Trincomalee, Sri Lanka

\begin{abstract}
Objective

To compare whether head and neck cancer treatment provided at the district general hospital in Trincomalee is within the international temporal guidelines and if not the necessary measurements needed for improvement.
\end{abstract}

\section{Methodology}

A Retrospective clinical audit of head and neck cancer care provided in 2016 and a re-audit in 2017 after application of remedial measures identified in the initial audit

\section{Result}

Patient delay, referral delay, initiationdelay, procedural delay, biopsy delay and treatment delay were minimized to the delays accepted by European guidelines.

\section{Conclusion}

Following the audit, the delays were identified involving all the stakeholders and implementation of an improvement plan done leading to near ideal head $\&$ neck cancer care provision.

Key words: audit, improvementplan, delay.

Copyright: () 2019 Ekanayake LSBet al.

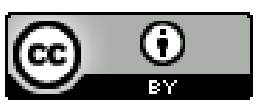

This is an open access article distributed under the Creative Commons Attribution License (CC-BY), This license lets others distribute, remix, tweak, and build upon the work, even commercially, as long as they credit the original author for the creation.

Funding: None

Competing interest: None

Correspondence:Ekanayake LSB(sapumal_ekanayake@yahoo.com)

iD https://orcid.org/0000-0002-1843-3299

Accepted Date : 12/2/2019

Published Date : 2/3/2019 


\section{Introduction}

As an ENT clinic in a developing country we wanted to know how efficiently our clinic delivers the recognized standard care to patients with head and neck cancers. We audited the care provided during the year2016.Our annual clinic turnover for the year was 9775and 23 patients had been diagnosed and treated for cancer of the head and neck during this period. The audit was performed in January 2017 and re-audited in December 2017, with the aim of discovering, whether the time from first presentation to initiation of cancer treatment, fell within internationally accepted time periods, by comparing with Minimum Temporal Standards of head and neck cancer care declared in the Third Consensus Document by British Association of Otorhinolaryngologists Head and Neck Surgeons ${ }^{[1]}$ and if it falls short of the expectation, how we can improve.

\section{Method and Sample}

Using clinic records, all of the patients who were diagnosed with head and neck cancer and treated (incidence) during the year 2016 wereanalysedretrospectively. The patients whoattended follow up visits, who presented with recurrence and who had been actively treated during the period but,who initially presented in the year 2016 (incidence)were included.

All the patients were traced from the clinic registry and interviewed by the consultant for

1. Details of the period of symptoms(Patient Delay).

2. Time laps between first visit to GP/ Primary Health care providerand first visit to ENT consultant(Referral Delay).

3. Timelapses in initiating procedures since the first visit to ENT clinic including Indirect Laryngoscopy (IDL),Fibre optic laryngoscopy (FOL), Flexible upper gastrointestinal endoscopy(UGIE), Fine needle aspiration cytology(FNAC), incision and excision biopsy, Direct laryngoscopy (DL), Rigid Oesophagoscopy, Ultra sound scan(USS) and Computerized tomography CT). (Initiation Delay.)

4. Time laps in execution of the above planned procedures.(Procedural Delay).

5. Time taken to arrive in diagnosis after performing the above procedures, (Biopsy Delay).

6. Time taken for surgical curative/palliative treatment execution from the first date of diagnosis.

7. Time taken from the first date in clinic to first date in registration of chemo or radiotherapy by oncologist.

The data collection was done by interviews with patients, analysis of clinic books and record books in the histopathology and radiology department and entered in to a Microsoft excel spreadsheet. In data collection and analysis of records, we came across many shortcomings in record keeping including incomplete data entry, missing pagesin clinic books, poor hand writing specially in relation to dates. Furthermore, clinic books had lot of wear and tear after travelling between institutes. Moreover, the interviews became difficult due to the language problem as many did not speak English or Sinhala, poor education level and difficulty in speech due to radiation. In addition, the data collection and adhering to audit criteria became a challenge as the medical officers, nurses and minor staff kept on changing and informing them of the importance and the ongoing procedure of the audit was difficult.

\section{Results}

\section{Patient Delay}

Time taken for a patient to seek a medical opinion from a GP or a specialist for the first time for the current diagnosis (patient delay)of cancer on average was 106.54 days. The minimum was 4 days while the 
maximum was 730 days. Median was 45 days. Only $33.3 \%$ had looked for medical help in the standard time of 4 weeks.

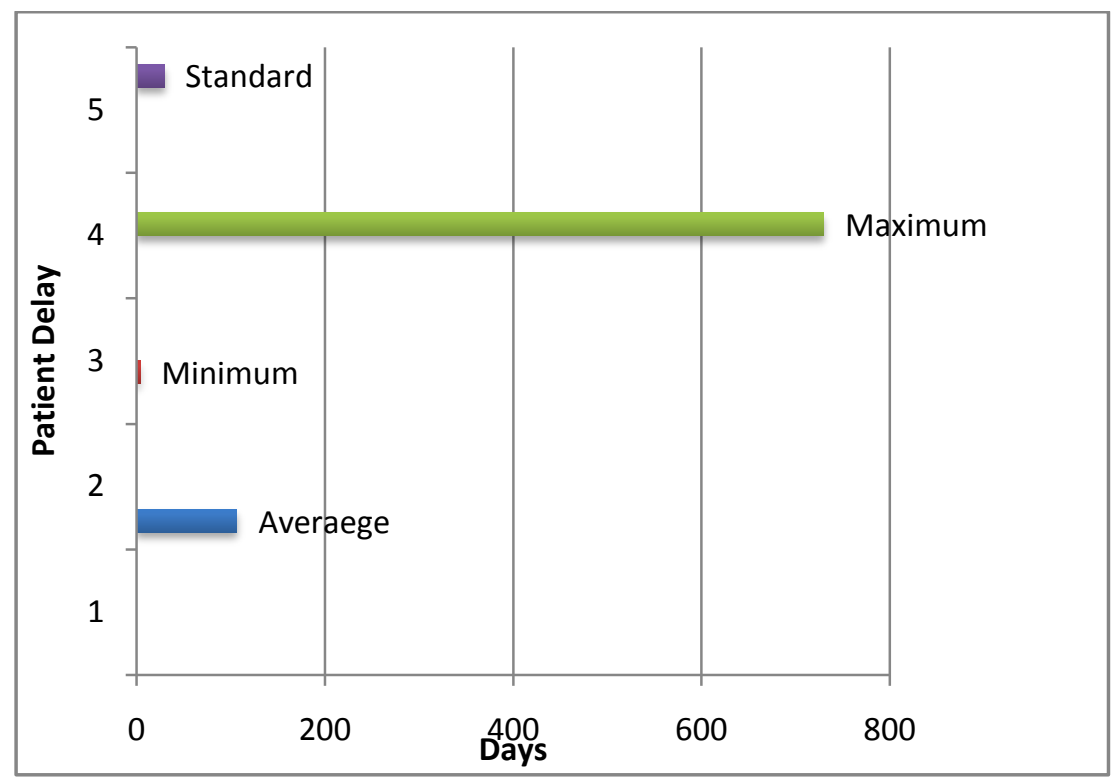

Figure 1

\section{First Specialist Appointment(Referral Delay)}

The total number of patients who were first seen by a GP or seen in the OPD and referred to the ENT clinic was 12.11 out of that $12(91.66 \%)$ were seen by the consultant within 14 days from the referral was made. 10 patents walked into clinic without any referral and were seen on the same day. The mean time for first Specialist Appointment was 5.166 and the mode was 1.

\section{Delay in planning procedures.}

According to the standards selected, the planning of procedures has to be done on the day of the first visit. In our audit 7 patients under went indirect laryngoscopy (30.43\%) and all who under wentindirect laryngoscopy had the investigation planned on the first visit.23 patients had Fibre-optic laryngoscopy planned and 20of them had it arranged in the first visit( $86.95 \%)$.There were data of 19 patients who had planned to undergoUpper gastro intestinal endoscopy and 18 of them had it planned in the first visit (78.26\%)Direct laryngoscopy was performed in 16 patients and 10 had it planned on day one (43.47\%).Open biopsy under local anaesthesia was performed in7 and 6 patients had it planned on day one (85.71\%)Imaging was planned amongst 20 patients and only 14(60.86\%) had it planned it by the end of the first visit.

\section{Procedural Delay}

This is the time taken from planning of procedures to execution of them. Fibre-optic laryngoscopy was performed in 16 and 14 patients had it performed within 7 days $(87.5 \%)(7$ patients didn't undergo FOL, instead 4 underwent DL 4.1 vocal cord lesion patient had an Examination under Anaesthesia (EUA)following IDL consequently no FOL was performed.1 patient with a lesion of the Piriform fossa who presented with dysphagia had a UGIE and a DL (No details were found on why his FOL was not performed after planning).Upper gastro intestinal endoscopy was performed in16and $12(75 \%)$ had it performed in 7days (4 patients had direct laryngoscopy and rigid Oesophagoscopy instead of UGIE).6(85.7\%) out of 7 who had rigid Oesophagoscopy performed,had it in within 7days. For pan endoscopy the mean value was11.5 days and median value was 6 .

Ultra sound scans (USS) of abdomen, contrast enhanced Computerized tomograms of Neck and chest were used for diagnosis and staging of tumours. US scans of abdomen was performed in 13 and 7 had them done in7 days from the day of request (53.8\%). Ultra sound scanning was performed by doctors and due to heavy work load it must have been impossible to keep to the temporal standards. Out of 20CT scan requests 
10(50\%) were performed within the standard time period of seven days. (One patient defaulted and did not attend the CT scan, one patient went to Jaffna hospital after diagnosis of Piriform fossa cancer, and details of could not be found)

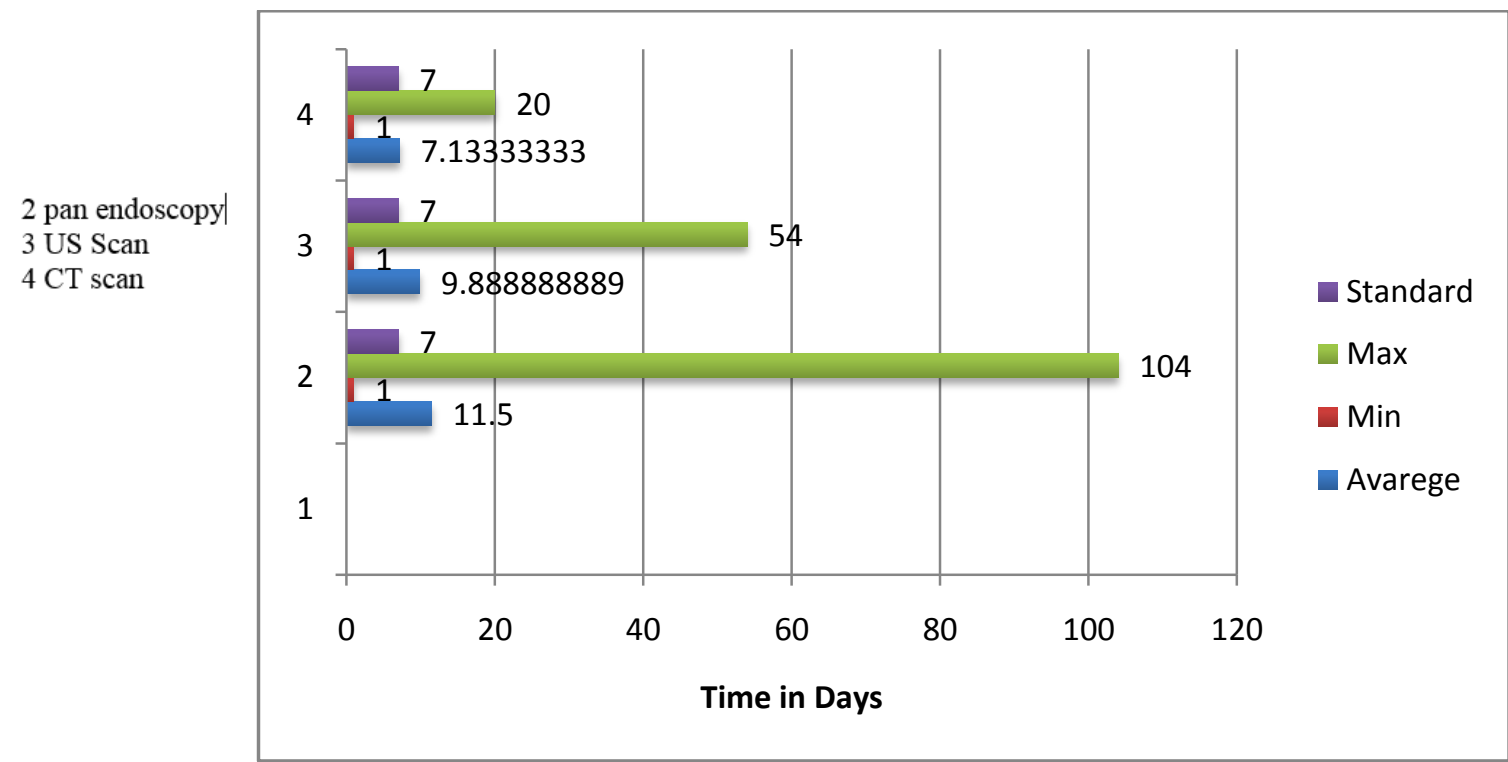

Figure 2: Procedural Delay

Arrival of biopsy sample at the pathology lab should happen without any delay. But no sample had been ever sent soon after surgery $(0 \%)$. FNAC reporting time should be 30 minutes and we had no slides reported with in this time $(0 \%)$.

\section{Biopsy Delay}

There were details of 19 patients (reported biopsy specimens) who underwent biopsy under general /local anaesthesia and 6(31.57\%) had them reported in the standard 7 days. The mean value for a biopsy to be performed and the report to be seen for the first time at the clinic by an ENT doctor was 8.5days.(This does not reflect on the in efficiency of the department of histopathology, as the patient was held responsible for tracing his/her reports before his/her visit to clinic.

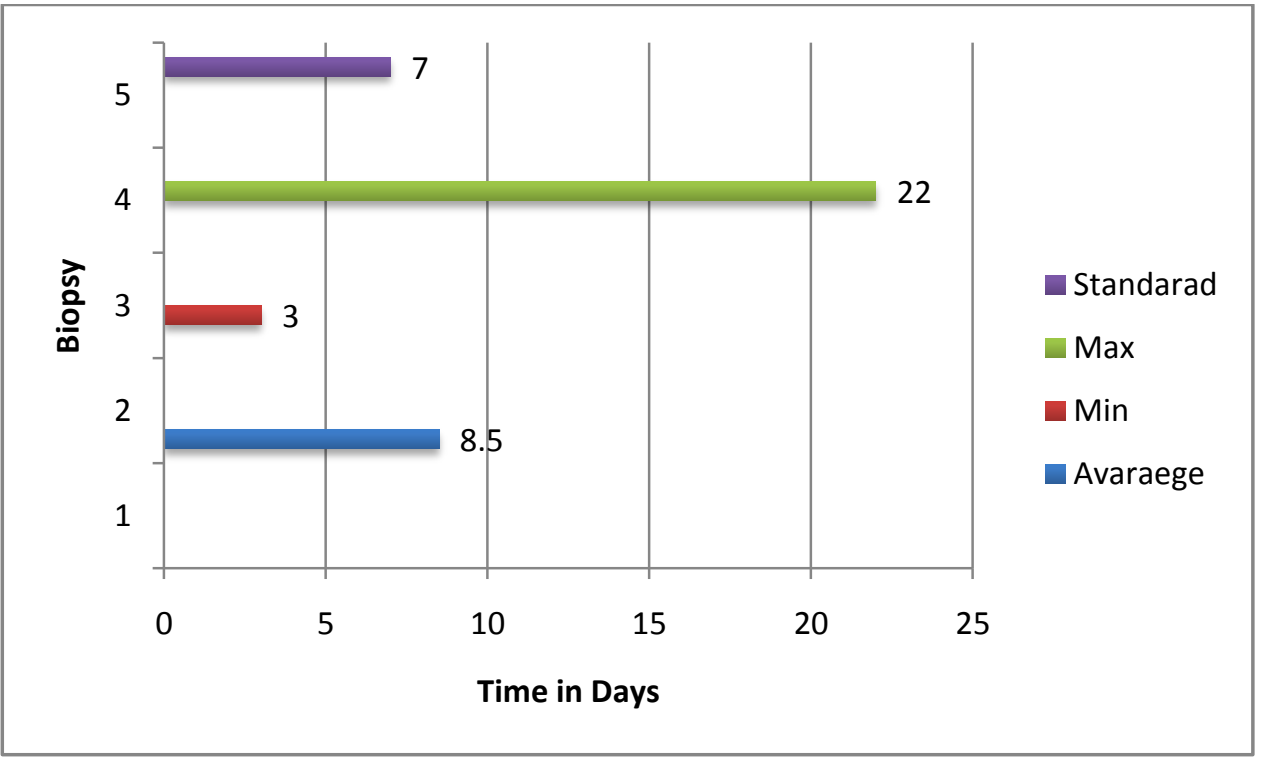

Figure 3: 


\section{Treatment Delay}

The time period for a patient to be referred after diagnosis from MHNC to radiotherapy /chemotherapy varies from 7 days and 14 days for palliative and curative care respectively ${ }^{[1]}$.In Sri Lanka we do not have MHNC and our patients, as a result after having a diagnosis of, Site, Staging and Histology, the otorhinolaryngologist directly refers them to the nearest oncologist. The oncologist decides the further management.

Below is arepresentation of the type and extent of lesions that were referred to oncology.

Table 1: The types, site and staging of head and neck tumours presented in one year.

\begin{tabular}{|c|c|c|c|c|}
\hline Site & Sub sites & Number & Staging & Cytology \\
\hline \multirow[t]{3}{*}{ Larynx } & Vocal Cords & 6 & $\begin{array}{l}\text { T1aM0N0 x3, } \\
\text { T3N0M0x1,T4aN3M)x1 }\end{array}$ & $\begin{array}{l}\text { Squamous Cell Carcinoma. } \\
\text { Well differentiated SCC, } \\
\text { Moderately Differentiated } \\
\text { SCC }\end{array}$ \\
\hline & Supraglottis & 5 & $\begin{array}{l}\text { T2bx1, } \\
\text { T3N2Bx1,T3N2M0x1,T4 } \\
\text { N0M0x2 }\end{array}$ & $\begin{array}{l}\text { Squamous Cell Carcinoma. } \\
\text { Well differentiated SCC, } \\
\text { Moderately Differentiated } \\
\text { SCC }\end{array}$ \\
\hline & Sub glottis & & & \\
\hline \multirow[t]{3}{*}{ Pharynx } & Piriform Fossa & 2 & T2N0M0x1,TxNxMx, & Poorly Differentiated SCC \\
\hline & Post cricoid & 3 & $\begin{array}{l}\text { TxNxMx,T4aN0M0x1,T4 } \\
\text { bM0N0x1 }\end{array}$ & $\begin{array}{l}\text { Well Differentiated } \\
\text { SCC,Moderately } \\
\text { Differentiated SCC, Poorly } \\
\text { Differentiated SCC }\end{array}$ \\
\hline & $\begin{array}{l}\text { Lateral Pharyngeal } \\
\text { Wall }\end{array}$ & & & \\
\hline Maxilla & & 2 & T4bN0M0 x1.T3N0M0 & $\begin{array}{l}\text { Small cell cancer } \\
\text { SCC }\end{array}$ \\
\hline \multirow[t]{3}{*}{ Oropharynx } & Tonsil & 1 & T3N2M0 & Well Differentiated SCC \\
\hline & $\begin{array}{l}\text { Posterior } 1 / 3 \text { of } \\
\text { tongue }\end{array}$ & 1 & T3N2 M0 & $\begin{array}{l}\text { Moderately Differentiated } \\
\text { SCC }\end{array}$ \\
\hline & $\begin{array}{l}\text { Posterior } \\
\text { Pharyngeal wall }\end{array}$ & 2 & $\begin{array}{l}\text { T3NOM0, } \\
\text { T2NOMO }\end{array}$ & $\begin{array}{l}\text { Moderately Differentiated } \\
\text { SCC } \\
\text { Poorly differentiated SCC }\end{array}$ \\
\hline Oesophagus & Mid1/3 & 1 & TxNxMx & SCC \\
\hline
\end{tabular}

Patients needing direct oncology referral were 17 and only 5patients had been referred within 14 days since the diagnosis was made. 2 patients fell of the radar as one Piriform fossa cancer left to another station(Jaffna)and 1 cancer of tonsil(T1) defaulted and came in 2017.4 patients had ablative surgery and $3(75 \%)$ had it performed within 14 days $(2$ modified radical cervical block dissections and one medial and anterior/lateral maxillectomy). 3 palliative surgeries were performed and they included two tracheostomies and one feeding gastrostomy with in 1 week of diagnosis. Out of 23 patients we could not find details of 3 patients about their referrals to oncologist and out of the rest,10 patients were referred to oncologist within 30 days and mean value was 43.78 days and median was 30 days. We were able to trace six patients who had documented evidence of the start of chemo/radiation and the mean value for initiation was 14.33 days from the referral to oncologist. There were no data available about follow up or rehabilitation following oncology or surgical intervention of these patients

\section{Discussion}


The mean time for a patient to seek any type of medical consultation primarily for the cancer concerned (Patient delay) was on average 106.54 days. This figure (our mean patient delay) as well as the median of 45 days may not be the ideal, which exceeds the standard of 4 weeks. According to literature patient delay may or may not play a major role in prognosis. This may become a concern as patient with a patient delay of 2 months or more have a significantly higher relative hazard of death according to Olli-PekkaAlho et al ${ }^{[2]}$.Overall, pharyngeal cancer patients who had a patient delay for 2 months or more had a 2.5 -fold risk for disease specific death ${ }^{[4]}$.Our patients prospect of survival may be influenced negatively by the delay in presentation. The main reasons for the delayed presentation amongst our study group, which may lead to poor prognosis; could be socioeconomic and of educational. Aquilina PJ who found his patients to have a mean patients delay of 106 days challenged these assumptions through a similar study and had demonstrated no relationship associated with socioeconomics, knowledge or geography with the patient's delay ${ }^{[3]}$. However, contradicting the latter in a study conducted by $\mathrm{Yu} T$ et al found median patient delay of 4.5 weeks, to be influenced vastly by geography and socioeconomics ${ }^{[6]}$.Abiding with the cause and result association of latter the south American study done by Andre Wady Debes Felippu et al found the median value of patient delay to be 10 months and one of the attributing factors being the geography ${ }^{[7]}$. Liewellyn et al defined patient delay as a delay of more than 3weeks while for Brouha et al it was a 30 days or a more period. In the research done by Pitchers and Martin it was more than 6 weeks ${ }^{[8]}$.In the context of worldwide deviation of the patient delay period, our median patient delay of 45 days is obviously not far behind though it does not follow the standards, which we are auditing our values against.

\section{Weakness andstrengths}

Increased patient delay time may be due to frank representation of affecting factors like socio-demography, socioeconomics as well as other factors like the small size of our sample. Secondly the diverse nature of our sample, such as laryngeal cancers which present early, to pharyngeal and maxillary sinus cancer which presented fairly late may also be another reason of our cancer patents mean value breaching the dead line. Most of the researches about patient delay were specific of the site and the sub site, resulting in calculating patient delay of a similar clinical presentation of a specific site, which vaguely would have similar duration of presentation. Most of the published audits were fairly focused of the site when it came to declaring in their patient delay, and rather than giving a single value for whole research they had being careful to give patient delay (period) variations for different sites. So many authors had taken the liberty of giving categorical values ${ }^{[8]}$.

The standard time for a patient to be seen by a specialist after the referral is made by GP is 14 days ${ }^{[1]}$. $91.99 \%$ of the GP referrals were seen within this time period in our clinic. When this exceeds 15 days it is called a referral delay ${ }^{[3]}$.In a study in Sarawak hospital in Malaysia where a similar health establishment exists they found the mean referral delay value of 8.4 weeks $^{[10]}$.Our Service provision in allocating a place for a suspected cancer patient with the specialist fell not only within our standards but also with in the accepted limits of other developed countries as New Zealand ${ }^{[5]}$.

The purpose of the analysis of the next section is to recognize which factors are linked with specialist delay and to establish the span of the diagnostic pathway in head and neck cancer patients. This section is broadly categorized as diagnostic delay. This is composed of procedure delay, imaging delay and biopsy delay.

\section{Procedure delay}

When analysing fibre-optic laryngoscopy and flexible upper gastro endoscopy statistics, rates of achieving the standards were $87.5 \%$ and $75 \%$ respectively, which is a significant positive figure. As far as Rigid Oesophagoscopy is concern, it was $85.7 \% \%$ as well. As the mean value for pan endoscopy was 11.5 days and median of 6 , these statistics fell far below the expected audit standards. Out of the 23 patients who underwent a diagnostic surgical procedure under general anaesthesia (DL,ML)10 had breached the temporal dead line, similarly Joel Franco MD et al, found delay in employing an appropriate diagnostic test or procedure in $(15.2 \%)$ which is approximately $1 / 3$ of ours ${ }^{[11]}$.

The reasons identified for the procedural delay were.

- Consultant being on leave and the solution taken to correct this was, all medical officers were trained to perform procedures(FOL,UGIE,IDL,Tonsillectomy) and to capture a video clip or series of still photographs during endoscopy to asses in diagnosis and to aid in staging and to attach the still photos to 
patient's records.

- Patients went missing after initial diagnostic procedure(FOL /IDL)and to avoid this a tool was introduced where all relevant contact details of patient and the closest relative is kept in clinic.

- FNAC results got delayed due to technical problems as slide contamination and all subsequent investigations that rely on FNAC had to be held back. Currently we get our FNAC in 24 hours minimizing the Domino effect of the delay in FNAC.

- Limitation of routine operating time.We have only one routine theatre list and due to the limiting time available for the consultant to attend in random times apart from the Monday list patients had to be kept minimally one week or more for a procedure under anaesthesia. To minimize procedural delay and avoid compromising the other clinical commitments medical officers were trained to perform procedures in the absence of consultant.

- Consultant fell sick.No procedure was attempted in the physical absences of the consultant to prevent compromising of quality for speed.

- Patient met with RTA and got hospitalized and was unable to be tracked after initial commencement of diagnostic procedure.

- Defaulting for native treatment. To avoid this currently we are emphasizing on the importance of Allopathic interference of cancer.

- Assuming the participation in a procedure as the responsibility of the patient after booking. As the duty of the doctor does not end as the patient leaves the clinic, we made this as the consultant's responsibility and appointed the consultant as the dedicated doctor in charge of cancer care provision. This was targeted mainly in improving biopsy and imaging time delays

- Lack of operating slot. Now suspected cancer patients get priority in operating lists.

- Fast tracked patient on anti platelet. Now we refer any patient with comorbidities for consultant anaesthetist's opinion parallel to initiation of other procedures.

- Delay in transporting specimen to lab, Fixing and cutting of specimens were done as an early procedure of the day hence if specimens do not reach the lab on time where processing gets delayed. With the aim of preventing this, the suspected cancer patients were operated as the first case and to prevent transport delay the specimen transport was handed over to the operating surgeon.

\section{Imaging Delay}

The mean value of US scan and CT scan delay were 9.8and 7.1 days. Only $58.8 \%$ and $53.8 \%$ of US scans and CT scans had met the 7-day standard respectively. This may appear as an underachievement though, even some of the developed countries have a lengthier time for imaging due to the technical difficulties. e.g. in New Zealand cancer guide line standards for imaging time is 14 days ${ }^{[5]}$ and sometimes the actual value was 18 days in other parts of developed world ${ }^{[13]}$.

\section{Biopsy delay}

The time taken from a biopsy to be reported from the day it is done, the median value was 7.9 and the mean was 8.5 days. The standard for this purpose was 7 days. Despite many short comingsthe services rendered by the pathology department is commendable especially in comparison with some studies from developed countries where mean and median were 62 and 21 days respectively ${ }^{[3]}$.Patel UA et al had similar findings where they took 14 days for diagnosis ${ }^{[13]}$.In Finland using an audit of three hundred and six patients the duration of the diagnostic process in a general hospital ranged from 0 to 570 days, with a median of 14 days, with only a small group of patients meeting the ideal management standards ${ }^{[9]}$.

In a publication from Malaysia a prospective study found the time from first consultation to histopathological diagnosis was 18.8 days $^{[10]}$.In another study the from Canada the median physician delay and patient delay were 108 and 31 days, respectively ${ }^{[12]}$.

We approached this with a quality improvement plan and implemented it when and where our care fell below the standard. The measures are discussed under the subtopic of discussion in detail. Following the audit, we managed to re-audit our performances in year 2017.

During our re-auditing cycle in 2017 we audited 7 randomly selected patients and below are the improved findings. Surprisingly the mean patient delay had become28.28 days from 106 days without any active 
intervention. The mean referral delay had changed to 5.14 days from5.116days. Initiation delay for IDL was 0.285 days with 2 breaching 0 -day dead line, FOL the mean was 0.4 days with 2 patient breaches, UGIE the mean was 0.5 days, for DL it was 0.75 days, for FNAC it was 0.3 days, for Biopsy it was a mean 0.16 days. Planning of US Scan and CT were 7.75 and 7 days respectively. These new findings in comparison with the former translates in the following manner. Mean referral delay was 5.142 and $100 \%$ of the patients were seen with in standard time. In initiation of procedures the below mentioned were the new changes, IDL from $30.43 \%$ to $71 \%$, FOL $86.95 \%$ to $71 \%$ UGIE from 78.26 to $57 \%$, DL $43.47 \%$ to50\%Biopsy from $85.71 \%$ to $83 \%$, USScan $60.86 \%$ to $50 \%$.

Performance delay section showed some really impressive improvements. FOL Median patient delay was 1.166 days and the percentage improvement went from $87.5 \%$ to $100 \%$. UGIE the mean delay was 4.6 days, and percentage of achievement with in standard time dropped from $75 \%$ to $66 \%$.For DL the mean waiting time was 2.71 days and success rate improved 85.7 to $100 \%$. WE were unable get FNAC within 30 minutes and successes rate was $0 \%$ though we got our FNAC within 0.66 days. Performance of biopsy was $100 \%$ within standard 7 days and average time was 3.99 days. US scan and CT scan performance delays were on average 6.28 and 3.71 days respectively. The imaging performed within standard period improved to $85 \%$ from 53.80\% in US Scan and 50\% in CT scans. Biopsy delay in reporting was 3.99 days and our previous rate of getting reports in time was $31.50 \%$ and re-auditing showed this to be $100 \%$ at present.

With regard to treatment delay;28\% of patients had ablative surgical intervention in the form of Modified radical block dissection and the average time was 30 days from the first day consultation. Success of operating within the standard time was $50 \%$ as one patient had an oncological referral followed by a CT scan of neck and unavailability of a blood urea report due to lack of reagents in hospital with undiagnosed hypertension. Subsequently he was operated in48days of the first consultation. Palliative surgical interventions were carried in three patients and all were in 7 days. Direct oncology referral was done on $72 \%$ and all were seen within 30 days of first consultation with otorhinolaryngologists. The average time of total delay was 18.6 days, which is a vast improvement.

\section{Conclusion}

It is with a great sense of satisfaction that we summarize this audit report as it shows an immense improvement to the care given for Head and Neck cancer patients at our Hospital. (The improvements are summarized compared to pre audit figures.)

- Patient Delay improved from106.54 to28.28 days.

- Referral Delay improved from 5.166 to 5.14 days (percentage covered with in standard period improved from $91.99 \%$ to $100 \%$ )

- Initiation Delay, IDL mean delay was 0.285 days (percentage covered with in standard period dropped from $100 \%$ to $71 \%$ ), FOL mean delay was 0.4 days (percentage covered with in standard period dropped from $86.95 \%$ to $75 \%$ ), UGIE mean delay was 0.5 days(percentage covered with in standard period dropped from78.26\%to57\%), DL mean delay was 0.5days(percentage covered with in standard period improved from $43.47 \%$ to50\% ),FNAC mean delay was 0.3 days $(0 \%)$,USS mean delay was 7.75days(percentage covered with in standard period dropped from60.86\%to50\%),CT mean delay was 7 days, Biopsy mean delay was 0.16Days(percentage covered with in standard dropped from $85.71 \%$ to83\%).

- Procedural Delay, IDL (percentage covered with in standard period improved from improved from $30.75 \%$ to $71 \%$ ) FOL mean delay was 1.66 days(percentage covered with in standard period improved from $87.5 \%$ to $100 \%$ ), UGIE mean delay was4.6 days(percentage covered with in standard period dropped from $77 \%$ to66\%.) DL mean delay was 2.71 days (percentage covered with in standard period improved from $85.5 \%$ to $100 \%)$.FNAC mean delay was 0.66 days $(0 \%)$.

- Imaging Delay, US Scan mean delay was6.28 days (percentage covered with in standard period improved from $53.8 \%$ to $85 \%$ ), CT scan mean delay was 3.71 days(percentage covered with in standard period improved from $50 \%$ to $85 \%$ ).

- Biopsy Delay, Biopsy mean delay was3.99days(percentage covered with in standard period improved from $31.50 \%$ to $100 \%$ )

- Treatment delay, Oncology referral improved from $43.78 \%$ and a mean of 30days to a mean of 18.6 with 
cover of $100 \%$ in standard period.

\section{Recommendations}

Tracking down patient details were made difficult due to many reasons including incomplete data entry and unclear hand writing hence, with the aim of improving this, we improvised a paper template(hard copy) that is more clear and complete, to be filled and attached to the first page of clinic book with a summary of all details. Furthermore, to improve on the procedural delay, training requirements were carefully assessed and will be tailored according to the deficiencies of skills of medical officers. Moreover, the practice needs to be (set up) changed to become friendlier to suspected cancer patients. Last but not least we plan to re audit entire initiation delay and procedure delay of the UGIE section to find out the reason for then egative result and how the new suggestions would help the cancer care provision after implementing the recommendations.

\section{Quality improvement plan}

- To reduce the patient delay, we need to improve the public awareness of symptoms of head and neck cancer. Intervention suggested are health education via public health midwife and medical officer of health for general public, for hospital visitors the message is to be sent via relevant clinics especially OMF, Dental and ENT clinics by heath education, distribution of leaflets and dispersion of information by multimedia under the responsibility of nursing officers.

- To reduce initiation delays, the consultant has to see all first visits and to initiate relevant investigations in first visit. With aim of achieving this we suggest to have a high degree of suspicion and to over screen and to have a false positive screening rate. The consequential increase of workload to be accommodated by making this hospital into a two consultant ENT unit.

- To improve procedural delay more staff training, including that of nursing and medical officers by consultant and via the ministry. Purchasing of surgical instruments including Light sources, fibre optic cables, light carrier for laryngeal speculum, adult rigid oesophagoscope and a suitable forceps and microlaryngoscopy forceps.

- Biopsy processing, the performing doctor is held responsible for this. To increase efficiency, we suggest to increase the carder of medical officers for the relevant department by minimally another doctor.

- Imaging delay, all cancer patients to get scanning on priority basis by discussing with consultant radiologist. (Responsibility is with consultant ENT surgeon).Also, to reduce the burden on the single consultant radiologist by filling the second consultant post in radiology at the institute.

- To improve treatment decision making we need to have a Multi-disciplinary team (MDT) with a consultant ENT surgeon, radiologist, pathologist clinical oncologist, speech and language therapist and OMF surgeon.

- To get a nutritionist and clinical oncologist and to start an oncology unit.

- $\quad$ The Planned date for re-audit, is January 2019

\section{References}

1. Effective Head and Neck cancer management.Third Consensus Document 2002.British Association of Otorhinolaryngologists and Head and Neck Surgeons.Last name(s), initial(s) of the author(s) NB! in the same order as in the book. Publication year. Book title: subtitle. Publisher, place of publication. Total number of pages ( $\mathrm{pp})$

2. Koivunen,P.Rantala,N.Hyrynkangas,K.Jokinen,K.Alho,O-P.2002incidence and survival in patients with pharyngeal cancer in northern Finland Eur Arch Otorhinolaryngol259 : 543-546 DOI 10.1007/s00405002-0506-6

3. Aquilina ,P,J.. 2007, An Investigation into Patient \& Professional Delays in the Diagnosis of Head \& Neck Cancer University of Sydney,pp 4

4. The impact of patient and professional diagnostic delays on survival in pharyngeal cancerAuthors.PetriKoivunenhttps://www.ncbi.nlm.nih.gov/pubmed/11753962 
5. Standards ofService Provision for Head and Neck Cancer Patients in New Zealand-Provisional

6. YU TI.WoodR,E.TenenbaunH,C.2008 Feb.2008 Feb.Delays in diagnosis of head and neck cancers.J Can Dent Assoc; $74(1): 61 .\{6=13\}$

7. 7.Felippu A,D,W . FreireE,C. ,R de Arruda Silva R.Guimaraes A,V.DeduvitisR,A. 2016, Impact of delay in the diagnosis and treatment of head and neck cancer.

8. CentellesP,V. Lopez-CodrunJ,L.Fernandez-SanromanJ.Seoane-RommeroJ,M.DeMelo $N$,S.AlvsrezNovoaP.GomezJ.Seoane J. 2017 January.Key points and time intervals for early diagnosis in symptomatic oral cancer: a systematic review. Oral and Maxillofacial Surgery.volume46,Issue 1.p1-10.

9. Brouha X,D. Tromp D,M. Koole R. Hordijk G,J. WinnubstJ,A.deLeeuw J,R. 2007 July.Professional delay in head and neck cancer patients: analysis of the diagnostic pathway.Oral Oncology .Volume43,issue6.p551-556.

10. Goy J.HallS,F.Feldman-Stewart D.Groome P,A. 2009 may.Diagnostic delays and disease stage in head and neck cancer:a systematic review.Laryngoscope. 2009 May;119(5):889-98. doi: 10.1002/lary.20185.

11. Franco, J. Elghouche N, A. Harris, M,S.Koloska M,S.2016 March.Diagnostic Delays and Errors in Head and Neck Cancer Patients: Opportunities for Improvement .American journal of Medical Quality, Vol.32,3pp330-335.[11=12]

12. Lee J,J.DhepnorratL,C.Nyhof-YounglanJ.Witterick I. 2016 March. investigating Patient and Physician Delays in the Diagnosis of Head and Neck Cancers: a Canadian Perspective. Journal of Cancer Education.31(1)8-14.

Patel U,A. Brennan T,E . 2012 August .Disparities in head and neck cancer: Assessing delay in treatment initiation.Laryngoscope,122(8):p1756-1760. 